\section{E-017 ROBOTIC NEUROVASCULAR SURGERY: SINGLE CENTER EXPERIENCE AND REVIEW OF THE LITERATURE}

M Costa*, C Pierre, M Basamh, A Patel, S Monteith. Cerebrovascular Neurosurgery Department, Swedish Neurosciences Institute, Seattle, WA

10.1136/neurintsurg-2021-SNIS.113

Introduction Robotics could expand treatment of rapidly progressive pathologies such as acute ischemic stroke, with the potential to provide populations in need prompt access to neuro-endovascular procedures.

Methods Robotically-assisted (RA) neuro-endovascular procedures (RANPs) performed at our institution were retrospectively examined (RA-group, RG). A control group of manual neuro-endovascular procedures was selected (manual group, MG). Total operating room (OR) time, procedural time, contrast media use, fluoroscopy time, conversion from RA to manual control, procedural success, and complication rates were compared. A learning curve was identified.

Results Forty-one (41) RANPs were analyzed. Ages ranged from 20-82 y.o. Indications included diagnostic cerebral angiography (37), extracranial carotid artery stenting (3), and transverse sinus stent (1). Total OR time was longer in RG (median 86 vs. $71 \mathrm{~min}, \mathrm{p}<0.01$ ). Procedural time (median 56 vs. $45 \mathrm{~min}, \mathrm{p}=0.12$ ), fluoroscopy time (median 12 vs. $12 \mathrm{~min}, \mathrm{p}=0.69$ ) and contrast media usage (82 vs. $92 \mathrm{ml}, \mathrm{p}$ $=0.54$ ) were not significantly different. Patient radiation exposure was similar, considering similar fluoroscopy times. Radiation exposure and lead apron use were virtually absent for the main surgeon in RG. Procedural success was $83 \%$ and conversion from RA to manual control was $17 \%$ in RG. No treatment-related complications occurred. A learning curve showed that, after the fifth procedure, procedural times reduced and stabilized.

Conclusions This series may contribute to further demonstrating the safety and feasibility of RANPs. RANPs can potentially reduce radiation exposure and physical burden for health personnel, expand acute cerebrovascular treatment to underserved areas, and enhance telementoring. Prospective studies are necessary for results to be generalized.

Disclosures M. Costa: None. C. Pierre: None. M. Basamh: None. A. Patel: None. S. Monteith: None.

\section{E-018 A COMPARISON OF TWO AUTOMATED CTP ALGORITHMS FOR ESTIMATION OF CORE INFARCT}

${ }^{1} \mathrm{~J}$ Vargas*, ${ }^{2} \mathrm{~S}$ Moorhead, ${ }^{3} \mathrm{M}$ Chaudry, ${ }^{1} \mathrm{R}$ Turner, ${ }^{1} \mathrm{~A}$ Turk. ${ }^{1}$ Neurosurgery, Prisma Health Upstate, Greenville, SC; ${ }^{2}$ USC School of Medicine Greenville, Greenville, SC; ${ }^{3}$ Prisma Health - Upstate, Greenville, SC

10.1136/neurintsurg-2021-SNIS.114

Introduction Automated analysis of CT perfusion data has become an integral part of stroke triage. Many vendors calculate cerebral blood flow (rCBF) and time to maximum perfusion $(\mathrm{Tmax})$ in attempts to estimate core infarct, reported as $\mathrm{rCBF}<30 \%$, versus tissue at risk, reported as Tmax $>6$ seconds. We compare the algorithms of two widely available software platforms, iSchemaView Rapid and Viz.AI.

Methods All patients on whom CT perfusion imaging for the suspicion of acute ischemic stroke was performed and also had subsequent underwent MRI imaging within 24 hours of CTP were included. As, sme patients underwent mechanical thrombectomy, $\mathrm{rCBF}<30 \%$ volumes were calculated to restrict analysis to core infarct. Both iSchemaView Rapid and Viz.AI software platforms were concurrently used. Final infarct volume was estimated off the B1000 sequence, in which areas of high signal were manually segmented using Slicer 3D.

Results 26 patients were identified. 14 underwent mechanical thrombectomy $(53.8 \%)$, of which 13 were recanalized to TICI $2 \mathrm{C}$ or better $(92.9 \%)$. $\mathrm{rCBF}<30 \%$ volumes calculated by both RAPID and Viz were to the segmented final infarct volume on MRI. The average differences was $30.73(+/-38.7) \mathrm{cc}$ for RAPID and $23.05(+/-39.7) \mathrm{cc}$ for Viz, which was not significantly different $(\mathrm{p}=0.515)$.

Conclusions There was not a statistically significant difference in calculated $\mathrm{rCBF}<30 \%$ volumes between Viz or Rapid, compared to the manually segmented volumes from the 24 hour diffusion MRI.

Disclosures J. Vargas: 2; C; Corindus, Medtronic, Cerenovus. 4; C; Visom, Ceretronic, Truvic. S. Moorhead: None. M. Chaudry: 2; C; Cerenovus, Medtronic, Q’Apel. 4; C; Q’Apel. R. Turner: 2; C; Siemens, Medtronic. 5; C; Siemens. A. Turk: 2; C; Siemens, Viz.AI, Medtronic. 4; C; Viz.AI.

\section{E-019 MECHANICAL THROMBECTOMY FOR NONAGENARIAN WITH M1 OCCLUSION: ANALYSIS FROM MULTICENTER COHORT}

${ }^{1} \mathrm{~S}$ Majidi*, ${ }^{2} \mathrm{~J}$ Vargas, ${ }^{3} \mathrm{~J}$ Blalock, ${ }^{4} \mathrm{H}$ Hawk, ${ }^{5} \mathrm{~S}$ Nimjee, ${ }^{5} \mathrm{~A}$ Zakeri, ${ }^{6} \mathrm{M}$ Mokin, ${ }^{7} \mathrm{R}$ Kellogg, ${ }^{8} \mathrm{G}$ Cortez, ${ }^{8} \mathrm{~A}$ Aghaebrahim, ${ }^{8} \mathrm{E}$ Sauvageau, ${ }^{8} \mathrm{R}$ Hanel, ${ }^{1} \mathrm{R}$ Deleacy, ${ }^{9} \mathrm{~A}$ Siddiqui, ${ }^{2} \mathrm{~A}$ Turk, ${ }^{10} \mathrm{M}$ Oselkin, ${ }^{10} \mathrm{E}$ Marlin, ${ }^{2} \mathrm{R}$ Turner, ${ }^{2} \mathrm{C}$ Chaudry, ${ }^{11} \mathrm{~J}$ Milburn. ${ }^{1}$ Neurosurgery, Mount Sinai Health System, New York, NY; ${ }^{2}$ Neurosurgery, Prisma Health - Upstate, Greenville, SC; ${ }^{3}$ USC School of Medicine Greenville, Greenville, SC; ${ }^{4}$ Radiology, Erlanger Health System, Chattanooga, TN; ${ }^{5}$ Neurosurgery, Ohio State University, Columbus, OH; ${ }^{6}$ Neurosurgery, University of South Florida, Tampa, FL; ${ }^{7}$ Neurosurgery, University of Virginia, Charlottesville, $V A ;{ }^{8}$ Neurosurgery, Baptist Medical Center, Jacksonville, FL; ${ }^{9}$ Neurosurgery, University of Buffalo, Williamsville, NY; ${ }^{10}$ St Luke's University Health Care, Bethlehem, PA; ${ }^{11}$ Radiology, Oschner Health, New Orleans, LA

\subsection{6/neurintsurg-2021-SNIS.115}

Introduction Elderly individuals are at an increased risk for stroke and are responsible for a substantial portion of the total stroke burden. The safety and efficacy of thrombectomy in nonagenarians is not fully understood, as they have traditionally been excluded from major endovascular trials. Recent literature suggests $7 \%$ of patients have functional independence at 3 months following medical management. This research aims to determine the outcomes of thrombectomy in patients $\geq 90$ years with proximal intracranial occlusion. 